\title{
Laparoscopic Longitudinal Pancreaticojejunostomy for Chronic Obstructive Pancreatitis
}

\author{
Eui Hyuk Chong, M.D., Jin Woo Lee, M.D., Sung Hoon Choi, M.D. \\ Department of Surgery, CHA Bundang Medical Center, CHA University, Seongnam, Korea
}

Purpose: Longitudinal pancreaticojejunostomy is a treatment option for selected patients with chronic obstructive pancreatitis that is rarely performed laparoscopically.

Methods: A 54-year-old man presented with chronic postprandial abdominal pain. A computed tomography scan revealed multiple calcified stones at the pancreatic head and tail with marked dilation of the pancreatic duct $(0.8 \mathrm{~cm}$ in diameter). The patient underwent laparoscopic longitudinal pancreticojejunostomy. Impacted stones were removed and Roux-en-Y pancreaticojejunostomy was performed using an intracorporeal suture technique in a longitudinal side-to-side manner.

Results: The total operation time and estimated blood loss were $150 \mathrm{~min}$ and $50 \mathrm{ml}$, respectively. The patient tolerated a regular diet without postprandial abdominal pain. Postoperative recovery was uneventful and the patient was discharged on postoperative day 5 in good condition. He returned to work on postoperative day 9.

Conclusion: Longitudinal pancreaticojejunostomy might be an effective option for relief of chronic pain in treating chronic obstructive pancreatitis.

Keywords: Laparoscopic, Minimally invasive surgery, Pancreaticojejunostomy, Chronic pancreatitis
Received July 26, 2017

Accepted August 16, 2017

Corresponding author

Sung Hoon Choi

Department of Surgery, CHA

Bundang Medical Center, CHA

University, Caner Research Building \#524, 59 Yatap-ro, Bundang-gu,

Seongnam 13496, Korea

Tel: +82-31-780-2990

Fax: +82-31-780-1824

E-mail: feel415@cha.ac.kr ORCID:

http://orcid.org/0000-0002-1664-3727

Supplementary video file: This article contains supplementary material (https://doi.org/10.7602/ jmis.2018.21.2.86).

This is an Open Access article distributed under the terms of the Creative Commons Attribution Non-Commercial License (http:// creativecommons.org/licenses/by-nc/4.0/) which permits unrestricted non-commercial use, distribution, and reproduction in any medium, provided the original work is properly cited.
Copyright (c) 2018 The Journal of Minimally Invasive Surgery. All rights reserved.

\section{INTRODUCTION}

Longitudinal pancreaticojejunostomy (LPJ) is a mainstay surgical option for patients with chronic obstructive pancreatitis in the presence of a dilated pancreatic duct and refractory pain when other treatments have failed. ${ }^{1,2}$ Laparoscopic LPJ is technically feasible and has comparable outcomes to the open approach. ${ }^{3,4}$ However, a laparoscopic approach for chronic pancreatitis is still in its infancy because of the complexity of this disease and the difficulty of the technique. The main purpose of this surgery is relief of chronic pain by resolving pancreatic ductal hypertension. Therefore, minimally invasive surgery may be advantageous for patients.

\section{MATERIALS AND METHODS}

A 54-year-old man presented with chronic postprandial abdominal pain. Computed tomography revealed multiple calcified stones at the pancreatic head and tail with marked dilation of the pancreatic duct measuring $0.8 \mathrm{~cm}$. He was not an alcoholic and the cause of chronic pancreatitis was idiopathic. A large impacted pancreatic head stone induced dilatation of 


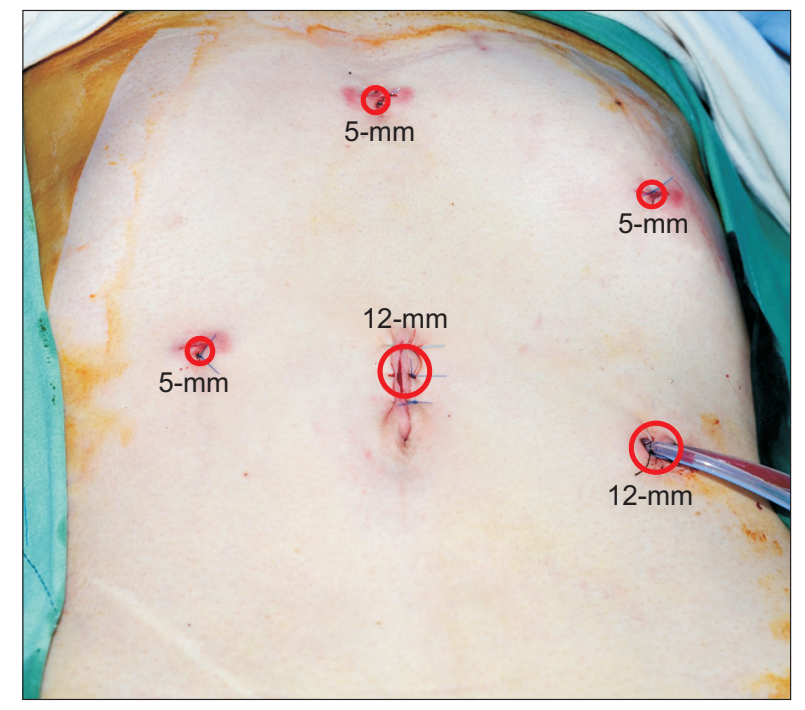

Fig. 1. Port placement. Two 12-mm trocars and three 5-mm trocars were used.

the pancreatic duct. Endoscopic retrograde cholangiopancreaticography and stenting of the pancreatic duct were attempted, but failed. Persistent pain and dilation of the pancreatic duct along with the presence of a main pancreatic duct stone could be indications for surgical intervention.

The patient was placed in the supine position with a headup tilt. Two 12-mm trocars and three 5-mm trocars were used (Fig. 1). Localization of the pancreatic duct was performed via intraoperative ultrasonography and the dilated pancreatic duct was opened longitudinally using a Thunderbeat (Olympus, Tokyo, Japan) and monopolar coagulator. Main pancreatic duct stones were removed. A Roux loop of the jejunum was harvested $20 \mathrm{~cm}$ distal to the Treitz ligament. The jejunum was divided using a 60-mm medium-thick endoGIA (Covidien Medtronic, St. Paul, MN, USA) and distal limb was placed along with opened pancreatic duct in retrocolic fashion. Pancreaticojejunostomy was performed using a $3-0 \mathrm{~V}-\mathrm{Loc}^{\mathrm{TM}}\left(\mathrm{Co}^{-}\right.$ vidien Medtronic, St. Paul, MN, USA) via an intracorporeal hand-sewn technique. Jejunojejunostomy was made $40 \mathrm{~cm}$ distal to the pancreaticojejunostomy site using a $60-\mathrm{mm}$ medium-thick endoGIA in a side-to-side manner. An indwelling drain was placed around the pancreaticojejunostomy.

\section{RESULTS}

The total operation time and estimated blood loss were $150 \mathrm{~min}$ and $50 \mathrm{ml}$, respectively. The patient resumed an oral diet on postoperative day 3 and diet was advanced as tolerated. The postoperative course was uneventful and the patient was discharged on postoperative day 5 in good condition. He returned to work on postoperative day 9. The first follow-up visit was 12 days after discharge, and the patient was tolerating a regular diet without postprandial abdominal pain.

\section{DISCUSSION}

Chronic obstructive calculous pancreatitis usually accompanies pancreatic duct hypertension, and is presumed to be the main pathophysiology. The goal of treatment for these patients is to ensure adequate drainage and decompression of the pancreatic duct in order to relieve the associated pain and preserve pancreatic exocrine and endocrine function. ${ }^{3}$ Of these patients, lack of response to other medical treatment or interventional modalities is the main indication for surgical intervention. Previous reports on LPJ have shown excellent postoperative outcomes in pain relief (75 94.3\%) with an acceptable minor complication rate $(11.8 \sim 30 \%)^{3,5,6}$ Although the main purpose of surgical intervention for chronic obstructive pancreatitis is pain relief, a minimally invasive approach has rarely been attempted., ${ }^{3,8}$

The major obstacles facing minimally invasive LPJ are the difficulty of removing impacted spiculated stones and long anastomosis. Recent experience of laparoscopic pancreatic surgery led proficiency in complex procedures, especially intracorporeal suturing. Fortunately, the texture of the pancreatic parenchyma is hard and main duct is large in chronic pancreatitis, enabling easy manipulation during anastomosis. A V-Loc absorbable barbed suture device allows easy and safe anastomosis and prevents loosening of the sutures. However, the pancreas spans the upper abdominal quadrants bilaterally. Therefore, in order to anastomize the long open pancreatic duct on both sides, the surgeon has to move, which is inconvenient. In addition, the spicules of main duct stones are frequently embedded in the branch duct and are not easily removed by laparoscopic instruments. It may be helpful to remove the stones directly using an open surgical long clamp through a small subxyphoid skin incision after removing the trocar.

LPJ might be an effective option for relief of chronic pain in chronic obstructive pancreatitis. A laparoscopic approach is technically demanding and time consuming due to the long anastomosis, but it can be performed safely thanks to accumulated experience. Such a minimally invasive approach would help to improve patient quality of life by minimizing treatment-related discomfort.

\section{REFERENCES}

1) Bachmann K, Izbicki JR, Yekebas EF. Chronic pancreatitis: modern surgical management. Langenbecks Arch Surg 2011;396:139- 
149.

2) D’Haese JG, Ceyhan GO, Demir IE, Tieftrunk E, Friess H. Treatment options in painful chronic pancreatitis: a systematic review. HPB (Oxford) 2014;16:512-521.

3) Tantia O, Jindal MK, Khanna S, Sen B. Laparoscopic lateral pancreaticojejunostomy: our experience of 17 cases. Surg Endosc 2004;18:1054-1057.

4) Palanivelu $C$, Shetty $R$, Jani $K$, et al. Laparoscopic lateral pancreaticojejunostomy: a new remedy for an old ailment. Surg Endosc 2006;20:458-461.

5) Adloff M, Schloegel M, Arnaud JP, Ollier JC. [Role of pancre- aticojejunostomy in the treatment of chronic pancreatitis. A study of 105 operated patients]. Chirurgie 1991;117:251-256; discussion 257.

6) Sielezneff I, Malouf A, Salle E, Brunet C, Thirion X, Sastre B. Long term results of lateral pancreaticojejunostomy for chronic alcoholic pancreatitis. Eur J Surg 2000;166:58-64.

7) Glaser C, Muller W, Zerz A, Szinicz G. [Laparoscopic laterolateral pancreaticojejunostomy]. Chirurg 2000;71:456-457.

8) Kurian MS, Gagner M. Laparoscopic side-to-side pancreaticojejunostomy (Partington-Rochelle) for chronic pancreatitis. J Hepatobiliary Pancreat Surg 1999;6:382-386. 